\title{
Conviviendo con una estoma húmedo: un estudio acerca de la calidad de vida
}

\section{Convivendo com uma estomia úmida: um estudo sobre qualidade de vida}

\section{Living with a wet colostomy: a study on quality of life}

Claudia Daniella Avelino Vasconcelos Benício ${ }^{1}$, Nalma Alexandra Rocha de Carvalho² , Isabela Ribeiro de Sá Guimarães Noleto 3 , Sara Machado Miranda ${ }^{4}$, Maria Helena Barros Araújo Luz

${ }^{1}$ Mestra em Enfermagem, docente de graduação da Universidade Federal do Piauí-UFPI

${ }^{2}$ Enfermeira pela Universidade Federal do Piaui-UFPI. Residente em Enfermagem Obstétrica- UFPI

${ }^{3}$ Enfermeira pela Universidade Federal do Piaui-UFPI

${ }^{4}$ Mestra em Enfermagem pela Universidade Federal do Piauí-UFPI

${ }^{5}$ Doutora em Enfermagem. Professora da Graduação e do Programa de Pós-Graduação Mestrado em Enfermagem da Universidade Federal do Piaui-UFPI.

Benício, C.D.A.V., Carvalho, N.A.R., Noleto, I.R.S.G., Miranda, S.M., \& Luz, M.H.B.A.

Cómo citar este artículo en edición digital: Benício, C.D.A.V., Carvalho, N.A.R., Noleto, I.R.S.G., Miranda, S.M., \& Luz , M.H.B.A. (2016). Conviviendo con una estoma húmedo: un estudio acerca de la calidad de vida.

Cultura de los Cuidados (Edición digital), 20( 46). Disponible en: < http://dx.doi.org/10.14198/cuid.2016.46.16>

Correspondencia: Nalma Alexandra Rocha de Carvalho. Campus Universitário Ministro Petrônio Portela, Bairro Ininga, Bloco 12. Teresina, Piauí, Brasil. CEP 64.049-550. Telefone: (86)3234-1219.

Correo Electrónico: enf.nalma.carvalho@hotmail.com

Reido: 07/03/2016; Aceptado: 20/08/2016

Recibido: 6/08//2015; Aceptado: 10/03/2016

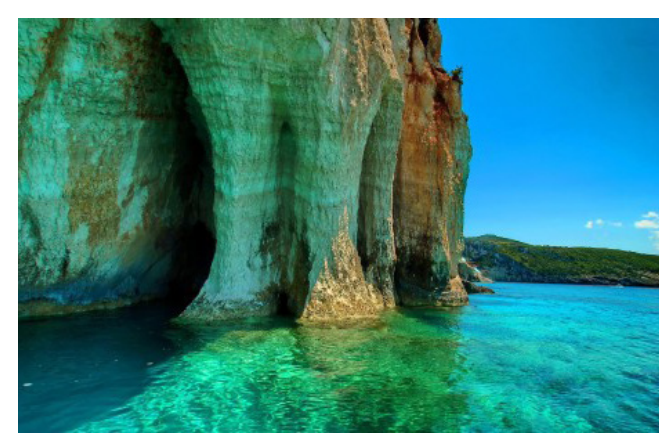

\section{ABSTRACT}

This study aimed to describe the sociodemographic profile and evaluate the life quality of a patient with permanent wet colostomy. It is a case study that deeply investigated the problems and needs of a patient with a wet colostomy and determined strategies to solve or ease the identified problems. The results were structured in related categories to sociodemographic data of the patient and the evaluation of their quality of life. The participant demonstrated to be well suited to the conditions to carry a wet colostomy. The acceptance of the colostomy patient with less than a year is a discontent. Those who already had the colostomy for many years can better adapt to the new condition. This study allowed to know and understand about the quality of life of a patient who has been living with a colostomy for many years, being able to offer valuable interventions to this public in later practices.

Keywords: Nursing. Quality of Life. Ostomy.

\section{RESUMEN}

Este estudio tuvo como objetivo trazar el perfil sociodemográfico y evaluar la calidad de vida de un paciente con ostomía húmeda permanente. Este es un estudio de caso que investigó a fondo los problemas y necesidades 
de una paciente con ostomía húmeda y ha determinado estrategias para resolver o mitigar los problemas identificados. Los resultados se han estructurado en categorías relacionadas con los datos sociodemográficos de la paciente y a la evaluación de su calidad de vida. La participante se mostró bien adaptada a las condiciones de portadora de ostomía húmeda. La aceptación del paciente portador de ostomía con menos de un año es de descontento, ya aquellos con el estoma durante varios años se pueden adaptar mejor a la nueva condición. El estudio posibilitó conocer y comprender acerca de la calidad de vida de una paciente durante muchos años con un estoma y por lo tanto puede proponer intervenciones valiosas a este público en sus prácticas posteriores.

Palabras clave: Enfermería. Calidad de Vida. Estoma.

\section{RESUMO}

Este estudo teve como objetivo traçar o perfil sociodemográfico e avaliar a qualidade de vida de uma paciente com estomia úmida permanente. Trata-se de um estudo de caso que investigou profundamente os problemas e necessidades de uma paciente portadora de estomia úmida e determinou estratégias para solucionar ou amenizar os problemas identificados. Os resultados foram estruturados em categorias relacionadas aos dados sociodemográficos da paciente e à avaliação de sua qualidade de vida. A participante mostrou-se bem adaptada às condições de portadora de estomia úmida. A aceitação do paciente ostomizado com menos de um ano é de descontentamento, já os que possuem o estoma há vários anos podem se adaptar melhor à nova condição. O estudo permitiu conhecer e entender a respeito da qualidade de vida de uma paciente há muitos anos com uma estomia, podendo assim propor intervenções valiosas a esse público nas suas práticas posteriores.

Palavras-chave: Enfermagem. Qualidade de Vida. Estomia.

\section{INTRODUÇÃO}

Estoma ou ostoma é uma palavra de origem grega que significa "boca" ou "abertura" utilizada para indicar a exteriorização de qualquer víscera oca no corpo. Assim tem-se a traqueostomia (abertura para a traqueia), gastrotomia (abertura para o estomago, para alimentação) e a urostomia, ilestomia e colostomia destinadas à eliminações (Silva, Figueiredo e Meirelles, 2009).

A colostomia úmida consiste na elaboração de um estoma a partir do cólon, onde a boca proximal se destina à eliminação de fezes e a boca distal à eliminação de urina (Ricci et al., 2009). A vantagem é que são eliminadas urina e fezes por uma única boca, mas em contrapartida é necessário encontrar um equipamento coletor que consiga adequar-se as duas drenagens (Habr-Gama e Araújo, 2000).

O Brasil possui cerca de 33.864 pessoas estomizadas (sem contar os Estados de Amapá, Tocantins e Roraima onde os números são desconhecidos); na região nordeste esse número chega a 4.176 e no Piauí aproximadamente 310 , número esse que vem crescendo a cada ano (Associação Brasileira de Ostomizados, 2008).

Pacientes submetidos a tal procedimento têm sua perspectiva de vida alterada, principalmente pela imagem corporal negativa, devido à presença do estoma associado a bolsa coletora. Além das mudanças nos padrões de eliminação, dos hábitos alimentares e de higiene precisam adaptar-se ao uso do equipamento, resultando em autoestima diminuída, 
sexualidade comprometida e, muitas vezes, em isolamento social (Nascimento et al., 2011).

Além de levar a grandes mudanças pessoais, sociais e emocionais, alterando, a auto-imagem e a auto-estima, podendo ocorrer estresse quando a pessoa se defronta com o novo problema ou a nova situação angustiante, respondendo com um temporário estado de desequilíbrio emocional. A sua adaptação e reação à nova imagem depende da capacidade emocional e física, variável em cada indivíduo que afeta significativamente a sua qualidade de vida (QV) (Camilleri-Brennan e Steele, 2002; Santos, Chaves e Kimura, 2006).

A compreensão sobre qualidade de vida lida envolve inúmeros campos do conhecimento humano, biológico, social, político, econômico, médico, entre outros, numa constante inter-relação. É necessário ter atenção à multiplicidade de questões que envolvem o universo da qualidade de vida, desde parâmetros sociais até de saúde ou econômicos. Esses indicadores podem ser analisados (e assim o são) por diferentes áreas de conhecimento, com referenciais e procedimentos diferentes, sendo vinculadas definições e concepções variadas (Almeida, Gutierrez e Marques, 2012).

Diante do contexto da complexidade e da problemática enfrentada pelas pessoas portadoras de estoma, este estudo teve como objetivo traçar o perfil sociodemográfico e avaliar a qualidade de vida de uma paciente com estomia úmida permanente

\section{METODOLOGIA}

Trata-se de pesquisa de abordagem qualitativa e o método escolhido foi o estudo de caso do tipo descritivo e de fundamento lógico-representativo (Yin, 2006). O estudo de caso, que apresenta como proposta metodológica com o intuito de investigar profundamen- te os problemas e necessidades do paciente, proporcionando a determinação de estratégias para solucionar ou amenizar os problemas identificados e, por último, avaliar o cuidado de enfermagem prestado (Galdeano, Rossi e Zago, 2003).

Foi realizado no período de agosto de 2014, em um Centro Integrado de Saúde Municipal de Teresina -PI, onde se desenvolve o único programa de dispensação de bolsas de estomia à comunidade do Estado. Na ocasião a paciente, sujeito deste trabalho, encontrava-se no referido Centro de Saúde juntamente com outras estomizadas para receber o seu estoque mensal de bolsas e mostrou-se receptiva ao diálogo, consentindo em participar como sujeito-alvo desta pesquisa.

Para a coleta dos dados foi elaborado um roteiro de perguntas semiestruturadas composto por perguntas referentes aos aspectos sociodemográficos. Utilizou-se ainda para a obtenção dos dados, o WHOQOL-BREF (World Health OrganizationQualityof Life - Breve), que é um instrumento genérico, desenvolvido pela Organização Mundial de Saúde (Organização Mundial de saúde, 2014). Instrumento curto e de rápida aplicação constituído por 26 perguntas, sendo as perguntas número 1 e 2 sobre a qualidade de vida geral e as 24 restantes compõem 4 domínios que são: físico, psicológico, relações sociais e meio ambiente. As respostas seguem uma escala de Likert, com itens de 1 a 5 , sendo que quanto maior a pontuação melhor a qualidade de vida.

Os aspectos éticos foram baseados nos preceitos da Resolução 466/12 do Conselho Nacional de Saúde (Brasil, 2012). Aprovado pelo Comitê de Ética em Pesquisa com Seres Humanos da Universidade Federal do Piauí-UFPI (Número do parecer: 302.522, CAAE $\mathrm{n}^{\circ}$ 10487813.3.00005214). A paciente mostrou 
sua aceitação em participar do estudo, por relato verbal e por meio da assinatura do Termo de Consentimento Livre e Esclarecido.

\section{RESULTADOS E DISCUSSÃO}

As categorias a seguir representam os dados sociodemográficos e a avaliação de sua qualidade de vida enquanto portadora de estomia úmida há mais de 20 anos.

\section{Dados Sociodemográficos}

Paciente H.A.O; 56 anos, do gênero feminino, divorciada, mãe de dois filhos, católica, com ensino superior completo e, atualmente, é aposentada. Mora em casa própria, com três pessoas residindo no domicílio, possui renda mensal familiar de 3(três) salários mínimos, boa convivência com os familiares e possui plano de saúde particular.

Quanto à idade e gênero, uma pesquisa realizada em um Centro de Referência para pessoas estomizadas, demonstrou que a maioria dos pacientes eram mulheres e a maior parte possuía entre 41 e 60 anos, eram aposentados e que a maior porcentagem tinha ensino fundamental incompleto. Nota-se que a participante deste estudo enquadra-se em boa parte do perfil deste estudo, discordando apenas quanto ao grau de instrução, pois a mesma possui ensino superior completo (Santos et al., 2007).

A causa base que levou à confecção do estoma de H.A.O. foi o diagnóstico e complicações de um câncer de reto. A paciente foi submetida tanto à colostomia quanto à ileostomia e cistectomia total. Seu estoma caracteriza-se como permanente, com exteriorização da alça terminal, que é característica de estomia permanente, exceto nas cirurgias de Hartmann. É localizado no quadrante superior esquerdo e elimina urina bem como fezes de característica pastosa.
A bolsa coletora é de duas peças específica para colostomia, aberta e possui barreira protetora de resina sintética. O estoma tem aproximadamente $25 \mathrm{~mm}$ de diâmetro, tem coloração rósea-avermelhada, é oval e apresenta uma pequena protrusão de $0,5 \mathrm{~cm}$. O abdome apresenta cicatrizes e sulcos que comprometem a aderência da bolsa, porém não provocando complicações de pele peri-estoma. A troca da bolsa é feita a cada 05(cinco) dias (Silva, Figueiredo e Meireles, 2007).

\section{Avaliação da qualidade de vida}

Utilizando o WHOOQOL-BREF pôde-se obter dados acerca da qualidade de vida da paciente, considerando-se as respostas obtidas para cada uma das questões que compõem os quatro domínios (físico, psicológico, social e meio ambiente) do questionário.

No domínio físico, observou-se que a entrevistada relata sofrer interferência pequena a moderada da dor para realização das atividades diárias, e o fato de ser estomizada não interferiu no padrão de sono, repouso e locomoção. Com relação às outras questões que compõem este domínio foi encontrado uma inferência moderada em relação a necessidade que ela tem de tratamento médico.

Quanto ao domínio psicológico a paciente relatou sentimentos que eram contemplados nas respostas médio e positivos com maior frequência. Afirmou estar satisfeita nas questões referentes ao aproveitamento e sentido da vida, capacidade de pensar, aparência física e realização pessoal. Atualmente, apresenta um bom nível de contentamento consigo satisfatório, visto que a preocupação com a ostomia já não é algo tão presente. A aceitação do paciente ostomizado com menos de um ano é de descontentamento, já os que possuem o estoma há vários anos se adaptaram a nova condição (Flek, 2005). 


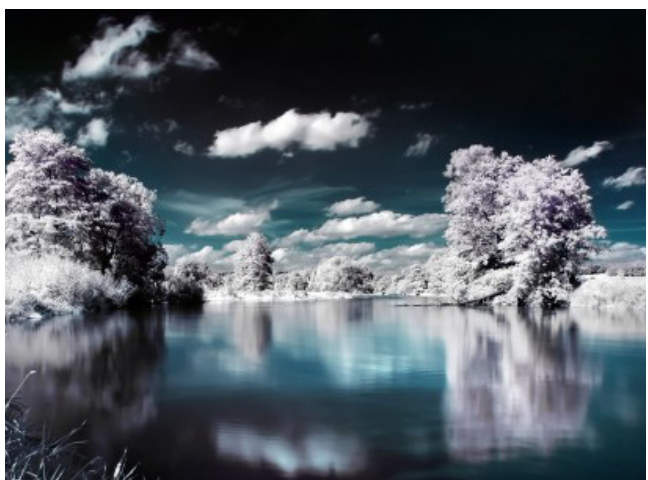

Com relação ao domínio de relacionamento social, a participantes revelou uma tendência maior à satisfação com as relações pessoais (amigos, família, conhecidos e colegas), além de demostrar satisfação quando se trata da sua sexualidade. A paciente mesmo estando divorciada, tem um parceiro fixo. A sexualidade parece ser afetada apenas quando o estoma é recente ou quando o paciente não possui parceiro fixo, pois se tornam inseguros, envergonhados com medo da aceitação ou não do parceiro (Bechara et al., 2005).

Já no domínio do meio ambiente, no que se refere à satisfação com os acessos ao serviço de saúde, a paciente optou por responder que não estava bem satisfeita nem insatisfeita. Porém, quanto à disponibilidade de informações diárias, condições de moradia e acesso aos meios de transporte, ela demostrou insatisfação. E nas questões referentes à segurança com vida diária, ambiente físico saudável, recursos financeiros suficientes e oportunidade de lazer, houve uma alternância de respostas entre nem satisfeito nem insatisfeito e insatisfeito.

No domínio do meio ambiente, apesar de se apresentar com os menores valores médios quando comparado aos outros domínios, foi o que apresentou maior elevação na média dos valores, o que não era esperado, já que neste período pós instalação da estomia, devido ao afastamento do trabalho remunerado as mulheres tendem a ter uma queda na renda financeira familiar, entretanto levando em consideração a alta porcentagem de mulheres que não tinham trabalho remunerado, pode-se deduzir que o processo de enfrentamento da doença leve as pacientes a uma maior aceitação de sua condição econômica.

\section{CONCLUSÃO}

A qualidade de vida de pessoas com estomia vem melhorando a cada dia, pois elas estão buscando independência tanto da vida pessoal quanto profissional. Verificou-se que os domínios mais comprometidos foram os domínios Social e Físico, e o mais preservado, o domínio Meio Ambiente. Portanto a ostomia não representa necessariamente o "fim da vida" desses pacientes, uma vez que poderão adquirir uma melhor qualidade de vida a partir do momento que participarem de atividades que lhes tragam prazer e motivação e que os conduzam a um viver em plenitude dentro de suas possibilidades. Esse estudo teve grande relevância para os autores pois permitiu conhecer e entender a respeito da qualidade de vida de uma paciente há tantos anos com uma estomia, podendo assim propor intervenções valiosas a esse público nas suas práticas posteriores.

\section{REFERÊNCIAS}

- Almeida, M.A.B., Gutierrez, G.L., \& Marques, R.(2012). Qualidade de vida. Definição, conceitos e interfaces com outras áreas de pesquisa. São Paulo: Escola de Artes, Ciências e Humanidades - EACH/USP, p.142.

- Associação Brasileira de Ostomizados- ABRASO.

(2008). Quantitativo aproximado de pessoas ostomizadas no Brasil. Disponível em: <http://www.abraso.org.br/ estatistica_ostomizados.htm $>$ acesso 30 de junho de $2014>$.

- Bechara, R.N., Bechara, M.S., Bechara, C.S., et al. 
(2005). Abordagem multidisciplinar do ostomizado.

Rev Bras Coloproct, 25 (2), 146-9.

- Camilleri-Brennan,J., \& Steele, R.J.C. (2002). Objective assessment of morbidity and quality of life after surgery for low rectal cancer. Colorectal Dis, 4 (1), 61-6.

- Fleck, M.P.A. (2000). O instrumento de avaliação da qualidade de vida da Organização Muncial da Saúde (WHOQOL-100): características e perspectivas. Ciênc Saúde Coletiva, 5 (1), 33-8.

- Galdeano, L.E., Rossi, L.A., Zago, M.M.F. (2003). Roteiro institucional para elaboração de um estudo de caso clínico. Rev Latino-AmEnferm, 11 (3), 371-5.

- Habr-Gama, A.E., Araújo, S.E.A. (2000). Estomas intestinais: aspectos conceituais e técnico. Assistência em estomaterapia: cuidando do ostomizado. São Paulo: Atheneu; 2000.

- Brasil. Ministério da Saúde (2012). Conselho Nacional de Saúde. Resolução 466/12 sobre pesquisa envolvendo seres humanos. Brasília: Ministério da saúde.

- Nascimento, C.M.S., Trindade, G.L.B., Luz, M.H.B.A. et al. (2011). Vivência do paciente estomizado: uma contribuição para a assistência de enfermagem. Texto
Contexto Enferm, 20 (3), 557-64

- Organização Mundial de Saúde -OMS. (2014). Versão em português dos instrumentos de avaliação qualidade de vida (WHOQOL). Disponível em: < http://www. ufrgs.br/psiq/whoqol.html>.

- Ricci, M.A., Duarte, E.L.C., Souza, R.C.A. et al. (2009). Hemicorporectomia associada à colostomia úmida: procedimento de exceção. Rev. Col. Bras. Cir, 36 (6), 25-528.

- Santos, C.H.M., Bezerra M.M., Bezerra F.M.M. et al. (2007). Perfil do Paciente Ostomizado e Complicações Relacionadas ao Estoma. Rev bras Coloproct, 27 (1), 016-019.

- Santos, V.L.C.G., Chaves, E.C. \& Kimura, M. (2006). Quality of life and coping of persons with temporary and permanent stomas. J WOCN. 33 (5), 503-9.

- Silva, R.C.L., Figueiredo, N.M.A. \& Meireles, I.B. (2009). Feridas: Fundamentos e Atualizações em Enfermagem. São Caetano do Sul: editora Yendis. - Yin, R.K. (2006). Estudo de caso: planejamento e métodos. $3^{\text {a }}$ ed. Porto Alegre (RS): Bookman. 